\title{
MAMLD1 and Differences/Disorders of Sex Development: An Update
}

\author{
Mami Miyado ${ }^{a}$ Maki Fukami ${ }^{a}$ Tsutomu Ogata ${ }^{a}$ b \\ aDepartment of Molecular Endocrinology, National Research Institute for Child Health and Development, Tokyo, \\ Japan; ${ }^{\text {DDepartment }}$ of Pediatrics, Hamamatsu University School of Medicine, Hamamatsu, Japan
}

\section{Keywords}

DSD · Mastermind like domain containing $1 \cdot$ Mutation ·

Oligogenic

\begin{abstract}
MAMLD1 (alias CXorf6) was first documented in 2006 as a causative gene of $46, X Y$ differences/disorders of sex development (DSD). MAMLD1/Mamld1 is expressed in the fetal testis and is predicted to enhance the expression of several Leydig cell-specific genes. To date, hemizygous MAMLD1 variants have been identified in multiple $46, X Y$ individuals with hypomasculinized external genitalia. Pathogenic MAMLD1 variants are likely to cause genital abnormalities at birth and are possibly associated with age-dependent deterioration of testicular function. In addition, some MAMLD1 variants have been identified in 46,XX individuals with ovarian dysfunction. However, recent studies have raised the possibility that MAMLD1 variants cause 46,XY DSD and ovarian dysfunction as oligogenic disorders. Unsolved issues regarding MAMLD1 include the association between MAMLD1 variants and $46, X X$ testicular DSD, gene-gene interactions in the development of MAMLD1-mediated DSD, and intracellular functions of MAMLD1.

(c) 2021 S. Karger AG, Basel
\end{abstract}

\section{Introduction}

MAMLD1 (Mastermind like domain containing 1; alias CXorf6, OMIM 300120) on the X chromosome is one of the causative genes for 46,XY differences/disorders of sex development (DSD) [Fukami et al., 2006]. The first MAMLD1 variants associated with 46,XY DSD were reported in 2006 [Fukami et al., 2006]. Subsequently, rare MAMLD1 variants were identified in several patients with 46,XY DSD and in a few patients with ovarian dysfunction [Kalfa et al., 2008, 2012; Chen et al., 2010; Brandão et al., 2011; Gaspari et al., 2011; Metwalley and Farghaly, 2012; Camats et al., 2015; Igarashi et al., 2015; Eggers et al., 2016; Ratan et al., 2016; Flück et al., 2019; Hughes et al., 2019; Li et al., 2020b]. MAMLD1 is assumed to play an important role in testicular function during the fetal period and after birth [Fukami et al., 2006; Kalfa et al., 2008, 2012; Camats et al., 2015; Igarashi et al., 2015; Fujisawa et al., 2016]. However, recent studies have shown that some $M A M L D 1$ variants are shared by unaffected individuals. It is possible that MAMLD1 variants lead to DSD in combination with other genetic abnormalities [Flück et al., 2019; Camats et al., 2020; Li et al., 2020a]. This review article aims to introduce the current karger@karger.com www.karger.com/sxd
(C) 2021 S. Karger AG, Basel

Karger"
Correspondence to:

Maki Fukami, fukami-m@ncchd.go.jp 
understanding of biological functions of MAMLD1 in sex development. We also refer to the results of animal studies on Mamld1.

\section{The MAMLD1/Mamld1 Gene}

\section{Chromosomal Position and Tissue Expression of}

MAMLD1/Mamld1

$M A M L D 1$ is located at $\mathrm{Xq} 28$, adjacent to MTM1 that represents a causative gene for myotubular myopathy [Tsai et al., 2005]. The etiological link between MAMLD1 and $46, \mathrm{XY}$ DSD was first suggested by the phenotype of patients with Xq28 microdeletions. Genetic male patients with these deletions frequently showed hypomasculinized external genitalia in addition to myopathy [Laporte et al., 1997; Ogata et al., 2008]. MAMLD1 was the sole gene located within the smallest region of overlap in 6 patients. MAMLD1 has no homolog on the Y chromosome, and its hemizygous pathogenic variants cause 46,XY DSD in an X-linked recessive fashion. In 46,XX individuals, MAMLD1 is predicted to undergo X chromosome inactivation [Nazor et al., 2012]. MAMLD1 is predominantly expressed in human gonads and is variably expressed in most other tissues, except for peripheral leukocytes (GTEx database). In particular, it is strongly expressed in the fetal testis [Fukami et al., 2006].

Murine Mamld1 is also located on the X chromosome [Miyado et al., 2015]. In situ hybridization analysis of mouse tissues revealed that Mamld 1 is clearly expressed in the Sertoli cells and in most of the Leydig cells of fetal testis [Fukami et al., 2006]. Quantitative PCR revealed that Mamld1 mRNA expression in the testis gradually increased from 12.5 to 18.5 days post coitum (dpc) [Miyado et al., 2012]. The expression pattern of Mamld 1 in fetal testicular tissues paralleled the increase in testosterone concentration. These findings support the possible role of MAMLD1 in testosterone biosynthesis. In situ hybridization showed that Mamld1 expression in the postnatal testis became relatively weak, while it was strongly expressed in the granulosa cells of the postnatal ovary [Fukami et al., 2006].

The expression of MAMLD1 in the testis is likely to be regulated by NR5A1 (alias SF-1/Ad4BP), a key molecule for testicular and adrenal development [Camats et al., 2020]. This notion is based on the previous findings that the MAMLD1 upstream region harbors a consensus binding motif of NR5A1 and that NR5A1 protein can bind to the sequence and exerts transactivation function [Fukami et al., 2008]. Baetens et al. [2017] have reported that MAMLD1 was upregulated in lymphocytes of patients with 46,XX tes- ticular/ovotesticular DSD due to the p.Arg92Trp variant of NR5A1. These data strongly suggest regulatory effects of NR5A1 on MAMLD1 expression. Other upstream regulators of $M A M L D 1$ remain to be determined.

\section{Human MAMLD1 Protein Structure}

The full-length MAMLD1 protein consists of 774 amino acids (NM_005491.4; NP_005482.2), while a naturally occurring splice variant lacking exon $4(\Delta$ exon 4$)$ creates a variant protein of 733 amino acids [Fukami et al., 2008]. The MAMLD1 proteins have structural similarity with the MAML2 (Mastermind like 2) protein, a non-DNA binding regulator of the Notch signaling [Fukami et al., 2008]. Both MAMLD1 and MAML2 contain a mastermind like motif and are capable of activating the promoter of Hes genes, particularly Hes3, in vitro. The full-length and $\Delta$ exon 4 forms of MAMLD1 exert the same in vitro transactivating activity on Hes promoters [Fukami et al., 2008]. Since Hes3 is involved in the regulation of cell differentiation and proliferation [Kageyama et al., 2005], this supports the possible role of MAMLD1 in embryonic development.

\section{In Vitro Function of Mamld1}

In vitro knockdown assays of Mamld 1 were performed using mouse Leydig tumor cells (MLTC1) [Nakamura et al., 2011]. Mamld 1 knockdown by 2 siRNAs significantly reduced the mRNA expression of Cyp17a1 and other genes involved in testosterone production. Consequently, the concentrations of testosterone, androstenedione, and dehydroepiandrosterone were significantly reduced in the culture media of Mamld 1 knockdown cells. In particular, these cells exhibited low testosterone concentration in the medium after $48 \mathrm{~h}$ incubation and after $1 \mathrm{~h}$ stimulation with human chorionic gonadotropin (hCG). Notably, the expression of Insl3 involved in testicular descent was also reduced, while the expression of Hes 3 remained unchanged. These data imply that Mamld 1 enhances testosterone production in Leydig cells primarily through the transactivation of Cyp17a1. Reduced expression of INSL3 may also contribute to 46,XY DSD. The results of these knockdown assays indicate that in vivo function of Mamld 1 is likely to be mediated independently of the Hes3-related Notch signaling pathway.

\section{Phenotypes of Mamld1 Knockout Mice}

\section{Mamld1 Knockout 40,XY Mice}

Miyado et al. [2012] created knockout mice of Mamld1. At birth, Mamld1 knockout 40,XY mice were healthy and 
exhibited a typical male genital appearance. Moreover, they showed no abnormalities in testicular histology, testicular weight, or immunohistochemical findings of several marker molecules in the testis. In addition, intra-testicular concentrations of testosterone and other steroids at $18.5 \mathrm{dpc}$ were as expected in normal males. On the other hand, fetal testes of the knockout mice were characterized by low mRNA levels of Leydig cell-specific genes such as Star, Cyp17a1, Hsd3b1, and Insl3, compared to the testes of wildtype mice. These results indicate that murine Mamld 1 is involved in the transactivation of several Leydig cell-specific genes in the fetal testis, although it is dispensable for genital masculinization.

In adulthood, Mamld 1 knockout 40,XY mice exhibited relatively small testes [Miyado et al., 2017]. In addition, reduced seminiferous tubule size, decreased number of proliferating spermatogonia and spermatocytes, and mildly impaired daily sperm production were observed. However, these mice exhibited no abnormalities in androgen levels in the serum or testicular tissues. Moreover, the number of pups born from cross-mated wildtype 40,XX mice was comparable between the knockout and wildtype 40,XY mice.

Altogether, these results imply that, in mice, MAMLD1 likely plays supporting roles in multiple steps of male sex development and reproduction. In particular, MAMLD1 appears to upregulate several genes in fetal Leydig cells. The lack of genital abnormalities in Mamld1 knockout mice indicates that there may be a difference in the functional importance of MAMLD1 between humans and mice. In this regard, although an Mamld1 variant has been identified in a horse with hypospadias, the pathogenicity of this variant remains uncertain [De Lorenzi et al., 2010]. In addition, Mamld 1 variants were similarly detected in genetic male dogs with and without DSD [Switonski et al., 2012]. Thus, further studies are necessary to clarify whether Mamld 1 variants affect male sex development in non-human species.

\section{Mamld1 Knockout 40,XX Mice}

Miyado et al. [2015] also examined the phenotype of Mamld1 knockout 40,XX mice. Although these mice were apparently healthy, they frequently exhibited parturition failure and resultant death of the pups. This phenotype can be ascribed to defective functional luteolysis. It is known that, in mice, the onset of parturition is triggered by a rapid decline in the blood level of progesterone due to functional luteolysis. The process of functional luteolysis depends on the enzymatic activity of $20 a$-hydroxysteroid dehydrogenase (20a-HSD) in the corpus luteum. MAMLD1 appears to be involved in the transactivation of Akr1c18, the gene encoding 20a-HSD, because ovaries of Mamld1 knockout 40,XX mice at late gestation were characterized by low expression levels of $A k r 1 c 18$. Notably, Stat5b, a suppressor of $A k r 1 c 18$, was significantly upregulated. Expression of other genes involved in the luteolytic process, such as Prlr and Oxtr, was also frequently dysregulated. These results indicate that in mice, MAMLD1 plays an indispensable role in labour initiation. MAMLD1 likely facilitates functional luteolysis by regulating the expression of $S t a t 5 b$ and other genes. Although it is known that circulating progesterone produced in luteal cells triggers the onset of parturition in several mammals, it remains unknown whether MAMLD1 is also involved in parturition of any species other than mice.

\section{MAMLD1 Variants in 46,XY Individuals}

\section{MAMLD1 Variants Identified in Patients with 46,XY DSD}

In 2006, 3 MAMLD1 nonsense variants, p.Glu197* (previously described as p.Glu124*), p.Gln270* (p.Gln197*), and p.Arg726* (p.Arg653*), were identified in 4 Japanese boys with 46,XY DSD [Fukami et al., 2006]. These boys manifested penoscrotal hypospadias with or without micropenis and cryptorchidism. Subsequently, hemizygous MAMLD1 variants were identified in patients with various types of 46,XY DSD [Kalfa et al., 2008, 2012; Camats et al., 2015; Igarashi et al., 2015; Flück et al., 2019]. MAMLD1 variants in the Human Gene Mutation Database include several protein-truncating substitutions and missense variants, as well as in-frame insertions and splice site variants (Fig. 1; Table 1) [Fukami et al., 2006; Kalfa et al., 2008, 2012; Chen et al., 2010; Ogata et al., 2012; Baxter et al., 2015; Camats et al., 2015; Igarashi et al., 2015; Ruiz-Arana et al., 2015; Eggers et al., 2016; Flück et al., 2019; Hughes et al., 2019].

We and other researchers performed in vitro assays to assess the function of MAMLD1 variants (Table 1). First, subcellular localization analyses were performed using fluorescence-tagged proteins [Fukami et al., 2008]. The results showed that the wildtype MAMLD1 exhibited speckled distribution in the nucleus and co-localized with MAML2. This speckled pattern was also observed for the p.Arg726* (p.Arg653*) variant, but not for the p.Glu197* (p.Glu124*) and p.Gln270* (p.Gln197*) variants. Second, luciferase assays using reporter vectors containing the promoters of Hes 3 , Hes7, or CYP17A 1 were performed to 


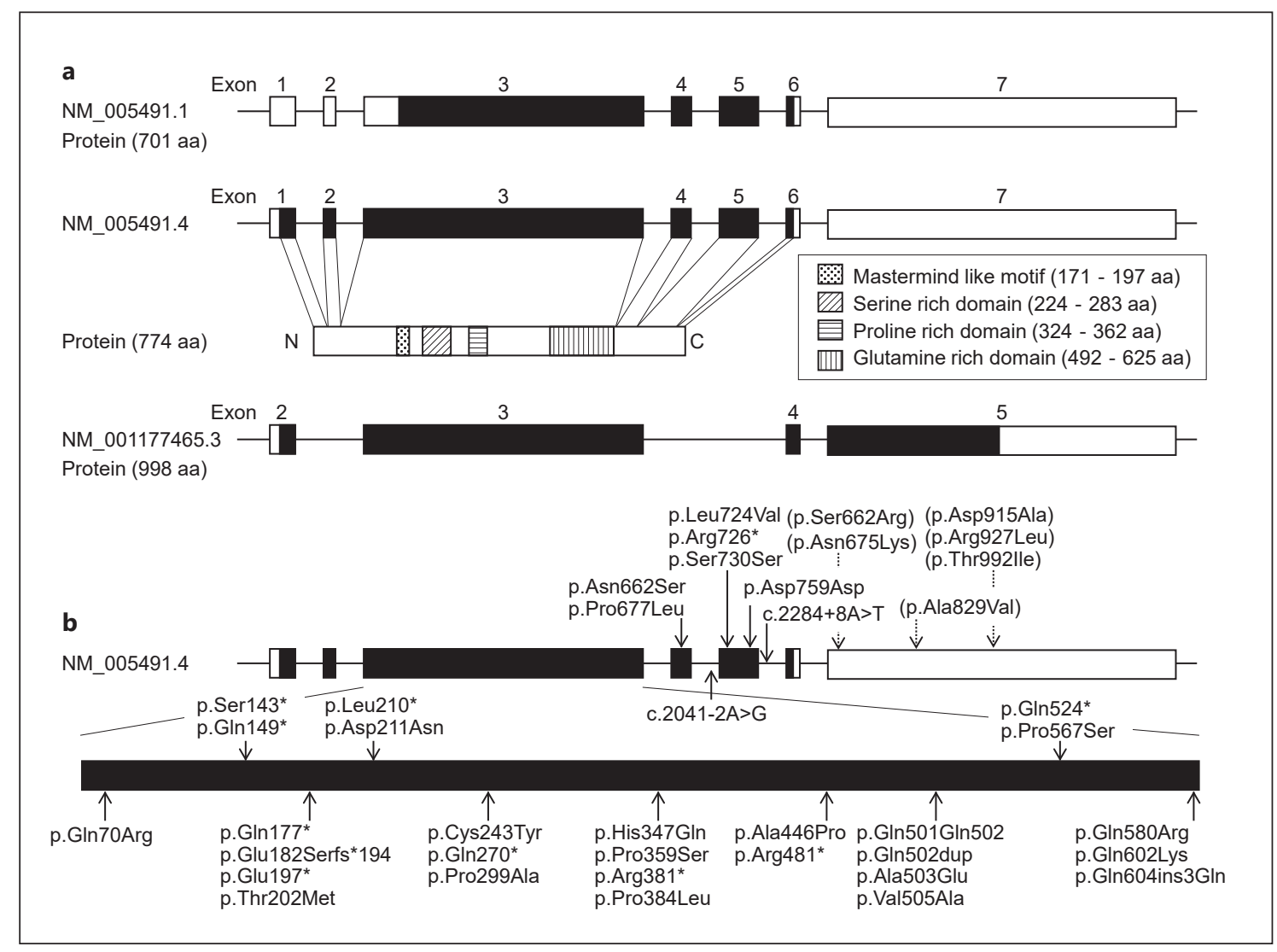

Fig. 1. Genomic structure of MAMLD1. a Structures of the human MAMLD1 gene (NM_005491.4 and NM_001177465.3) and its protein. The black and white boxes indicate the coding and noncoding exons, respectively. b Schematic representation of MAMLD1 variants described in Table 1. Solid and dashed arrows indicate the variants on NM_005491.4 and NM_001177465.3, respectively. The sizes of introns are not drawn to scale.

examine in vitro transactivating activities of MAMLD1 variants [Brandão et al., 2011; Camats et al., 2015]. The results showed compromised transactivating activities of several variants including a splice site variant. The $\Delta$ exon 4 variant was shown to retain normal activity. In this regard, some $M A M L D 1$ variants identified in patients with 46 ,XY DSD showed normal in vitro transactivating activity on the Hes 3 promoter [Fukami et al., 2008; Kalfa et al., 2011; Li et al., 2020b]. Thus, it remains unknown whether these variants are pathogenic mutations or benign polymorphisms. Indeed, these variants are classified into various functional categories according to the criteria of the American College of Medical Genetics and Genomics and the Association for Molecular Pathology [Richards et al., 2015] (Table 1). Third, protein expression analysis revealed reduced expression levels of various nonsense and splice site variants [Fukami et al., 2008; Camats et al., 2015; Igarashi et al., 2015]. Lastly, in silico analyses were performed to predict the effects of nucleotide substitutions on the 3-dimensional (3D) structure of the MAMLD1 protein [Kalfa et al., 2011]. These analyses predicted that some missense variants such as p.Pro567Ser (p.Pro542Ser) and p.Ser662Arg (p.Ser589Arg) lead to drastic changes in the interaction force of the amino acid chain and flexibility of the spatial structure.

\section{Phenotypes of Variant-Positive 46,XY Patients}

Hemizygous MAMLD1 variants have been identified in 46,XY individuals with micropenis, cryptorchidism, or complete gonadal dysgenesis [Fukami et al., 2006; Kalfa et al., 2008, 2012; Camats et al., 2015; Igarashi et al., 2015; Flück et al., 2019; Li et al., 2020b]. Li et al. [2020b] performed a review of their own 10 cases and previously reported 26 cases with $46, \mathrm{XY}$ DSD and rare MAMLD1 variants. The authors analyzed phenotypes of the patients and concluded that hypospadias is the most salient feature of 


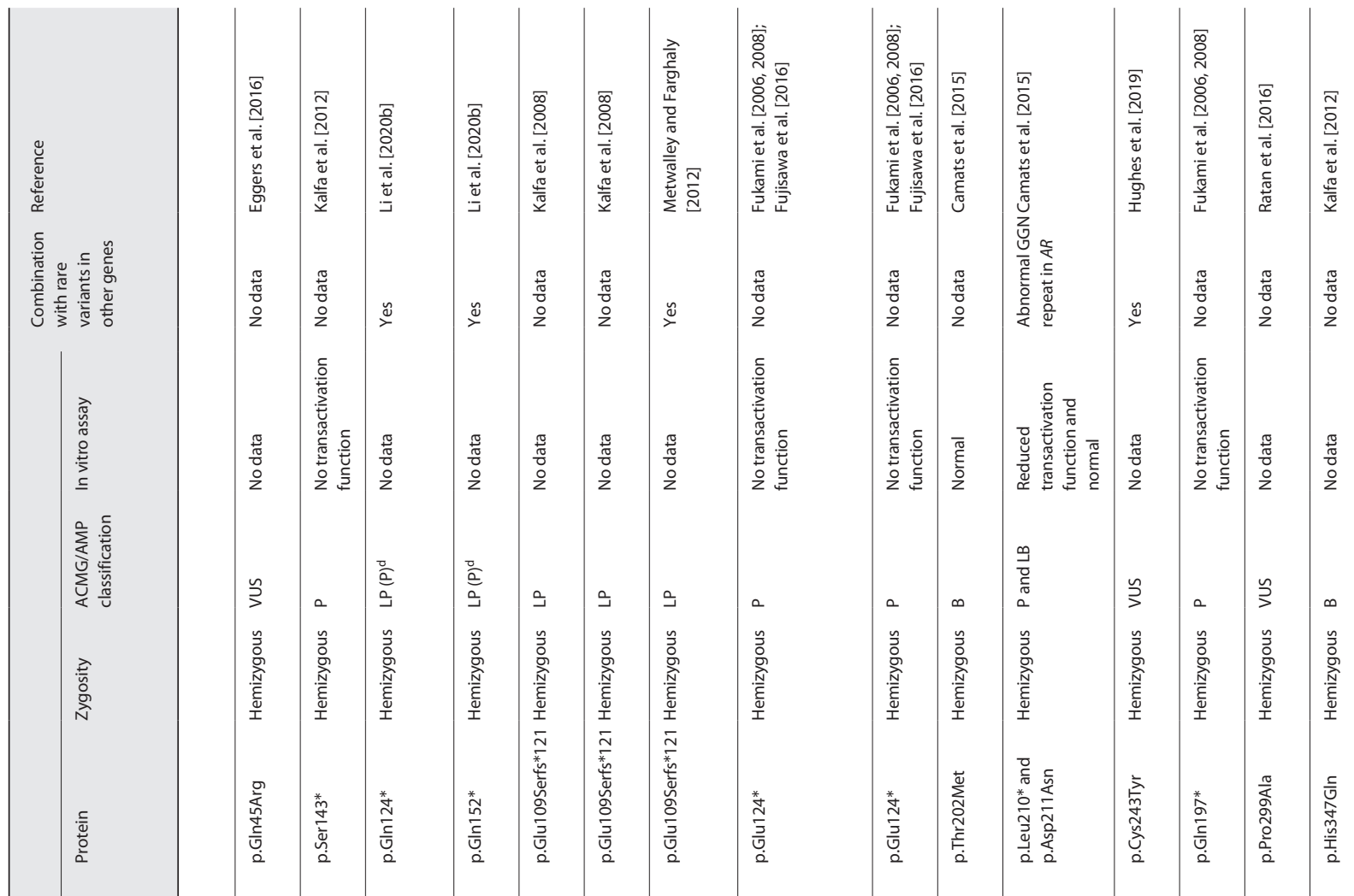






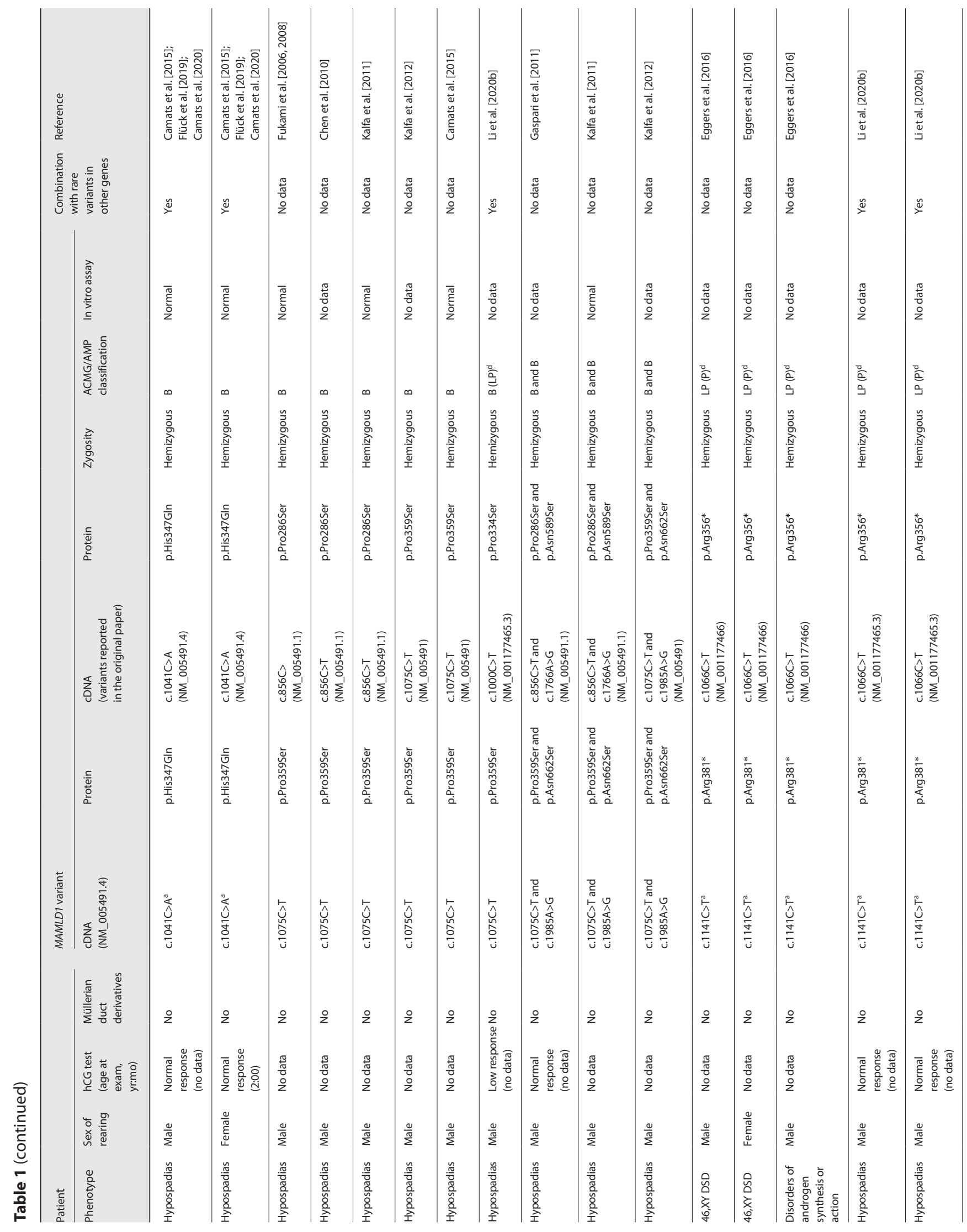




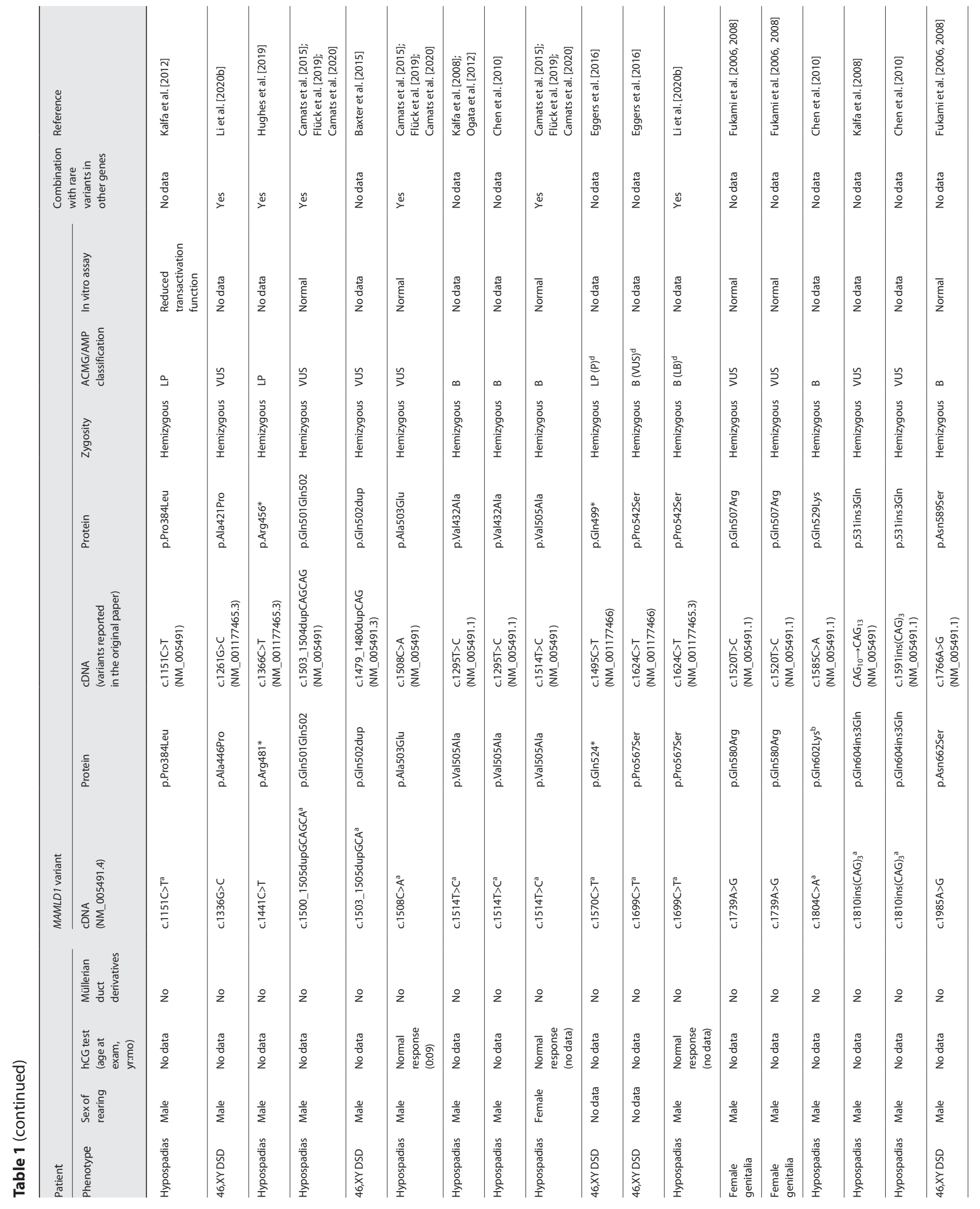




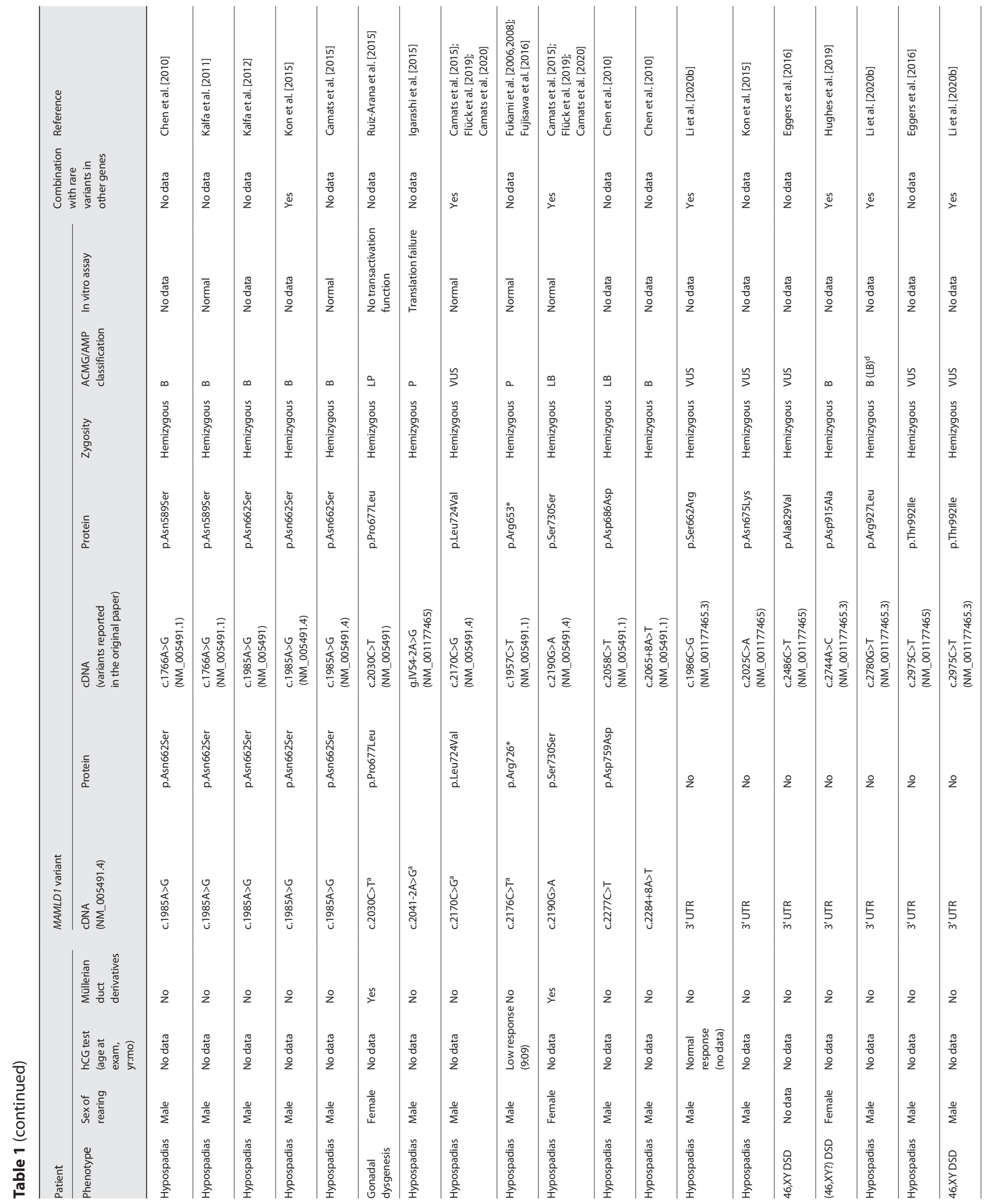




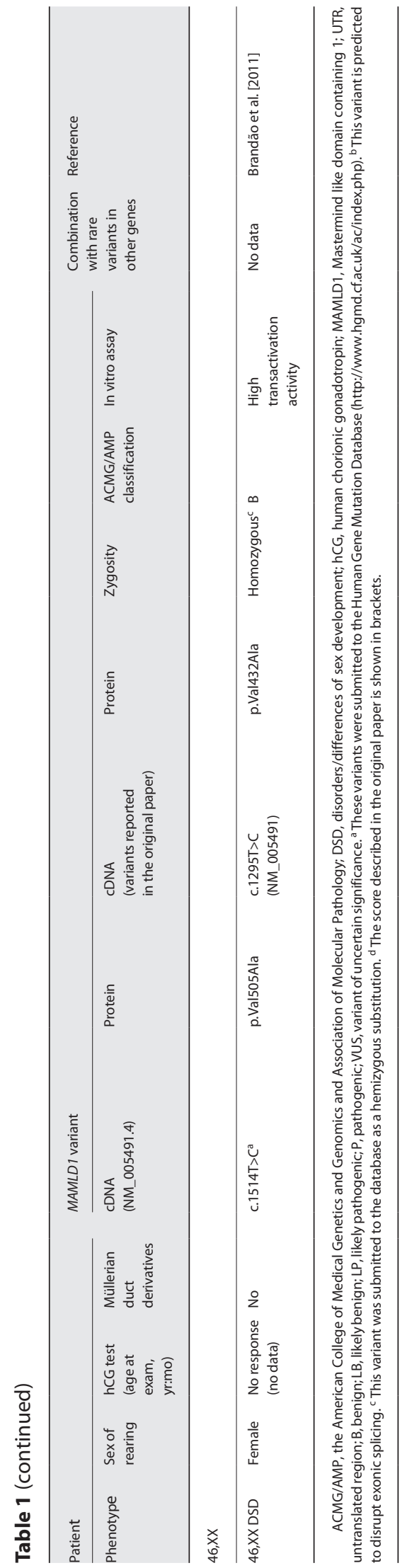

MAMLD1 abnormalities. The severity of hypospadias varied among the patients. In addition, micropenis was observed in about half of the cases and complete femaletype external genitalia was present in 4 cases. Adrenal dysfunction was not observed. Rare clinical features observed only in one or a few patients included craniofacial anomalies and precocious puberty. Only 1 patient had müllerian duct derivatives [Ruiz-Arana et al., 2015] (Table 1).

Most of the patients were subjected to blood hormone measurements during infancy. The levels of testosterone and gonadotropins were mostly within the normal range. Likewise, adrenal hormones were normal in all the patients examined. However, blood levels of $\mathrm{LH}$, and FSH were significantly elevated in 1 Chinese patient who exhibited precocious puberty [Li et al., 2020b].

The current understanding is that the major role of MAMLD1 during the fetal period is to enhance testosterone biosynthesis, and loss-of-function variants of MAMLD1 typically lead to partial hypomasculinization at birth. However, it remains unknown whether complete female-type external genitalia and precocious puberty identified in some patients are associated with the MAMLD1 variants. Furthermore, genotype-phenotype correlations of $M A M L D 1$ variants have yet to be studied.

\section{Long Term Follow-Up Study of Patients with 46,XY \\ DSD and MAMLD1 Variants}

Fujisawa et al. [2016] performed a longitudinal followup study of 3 patients with 46,XY DSD and hemizygous $M A M L D 1$ nonsense variants. At birth, these individuals exhibited penoscrotal hypospadias with chordee, micropenis, hypoplastic scrotum, and/or undescended/retractile testis. Blood levels of testosterone during infancy were within the normal range. However, these 3 patients invariably manifested impaired testicular function at later ages. One of the 3 patients (patient 1) manifested incomplete secondary sexual development together with micropenis and relatively small testes. The patient showed an inappropriate response of blood testosterone to the hCG stimulation. The remaining 2 patients (patients 2 and 3 ) also exhibited borderline micropenis at 7 and 6 years of age, respectively. Blood examinations of patients 2 and 3 at 7 and 9 years of age, respectively, suggested mild to moderate hypergonadotropic hypogonadism. In addition, ultrasound examinations revealed testicular microlithiasis, a non-specific sign of testicular dysfunction, in patients 1 and 3 . These results indicate that loss-of-function mutations of $M A M L D 1$ cause not only genital abnormalities at birth, but also age-dependent deterioration of 
testicular function. Consistent with this, delayed puberty indicative of partial hypogonadism was observed in a Swiss patient with a MAMLD1 variant [Li et al., 2020b]. On the other hand, a Chinese patient exhibited early puberty [Li et al., 2020b]. Thus, further studies are necessary to clarify the long term clinical features of patients with MAMLD1 mutations.

\section{Oligogenicity of MAMLD1 Variants}

As mentioned above, MAMLD1 variants were identified in a number of patients with 46,XY DSD. However, there is a debate about the functional significance of MAMLD1 in male sex development. Indeed, several findings, such as the identification of some $M A M L D 1$ variants in the general population, preserved in vitro transactivating activity of several DSD-associated MAMLD1 variants, phenotypic discrepancies among the patients with the same MAMLD1 variants, and the typical male genital appearance and preserved fertility of Mamld1 knockout 40,XY mice, pose a question as to whether single MAMLD1 variants are sufficient to cause 46,XY DSD [Fukami et al., 2008; Miyado et al., 2012, 2017; Camats et al., 2020].

Flück et al. [2019] raised the possibility that MAMLD1 variants lead to $46, \mathrm{XY}$ DSD when combined with rare variants in other genes. To test this hypothesis, the authors performed whole exome analysis for 7 patients with $46, \mathrm{XY}$ DSD and 1 patient with $46, \mathrm{XX}$ ovarian insufficiency, who harbored hemizygous and heterozygous $M A M L D 1$ variants, respectively. The authors analyzed the sequences of 606 genes that were possibly associated with MAMLD1-related signaling pathways or fetal sex development. As a result, Flück et al. [2019] identified 55 rare heterozygous/hemizygous variants in 41 genes that potentially contributed to the disease phenotypes of the 8 patients. For example, patient 1 harbored 9 rare nucleotide substitutions in 7 genes, in addition to the p.Val505Ala (p.Val432Ala) variant of MAMLD1. Most of the 7 genes have been associated with hypospadias and/ or syndromic genital anomalies. The remaining 7 patients also carried 4 or more rare variants in the tested genes. Most of the identified variants were classified into the categories of "variant of unknown significance (VUS)" or "likely-benign"; however, 2 variants were assessed as "likely pathogenic". These results support the oligogenic nature of MAMLD1-associated DSD. Since the first 3 patients with $M A M L D 1$ variants [Fukami et al., 2006] were not subjected to whole exome analysis, these individuals may also carry pathogenic variants in other genes. In this regard, systematic mutation screening for patients with
46,XY DSD have shown that a certain percentage of the patients harbored multiple variants in known causative genes [Kon et al., 2015; Eggers et al., 2016]. Thus, although there is no doubt that monogenic mutations account for a certain percentage of cases with 46,XY DSD, oligogenicity is likely to play an important role in the etiology of this disorder.

\section{MAMLD1 Variants in 46, XX Individuals}

Thus far, there are only a few reports of MAMLD1 variants in genetic females [Flück et al., 2019]. 46,XX individuals with heterozygous loss-of-function variants of $M A M L D 1$ appear to be phenotypically unremarkable, because the mothers of patients with 46,XY DSD and $M A M L D 1$ variants were reported to be healthy and fertile [Fukami et al., 2006; Kalfa et al., 2012; Ruiz-Arana et al., 2015]. However, considering the strong expression of MAMLD1/Mamld1 in the postnatal ovaries of humans and mice [Fukami et al., 2006; Miyado et al., 2015], MAMLD1 may play a role in ovarian function. Consistent with this, Flück et al. [2019] reported a 27-year-old Spanish woman with primary amenorrhea who carried a rare heterozygous variant in MAMLD1. The woman exhibited small ovaries with a large number of primordial follicles and atresic follicles. Endocrine examination confirmed hypergonadotropic hypogonadism. These data indicate that MAMLD1 possibly contributes to ovarian development or function. This notion is further supported by the report by Marozzi et al. [2000], in which the authors identified chromosomal deletion at Xq28 in a female with premature ovarian failure. In this regard, because the woman reported by Flück et al. [2019] harbored 14 additional variants in genes possibly associated with MAMLD1-related signaling pathways or fetal sex development, heterozygous MAMLD1 variants may cause ovarian dysfunction as an oligogenic disorder. Thus far, there have been no reports of homozygous loss-of-function variants of MAMLD1 in women. Thus, although homozygous deletion of Mamld 1 was shown to cause parturition failure in 40,XX mice [Miyado et al., 2015], it remains unknown whether complete loss-of-function of $M A M L D 1$ also leads to delayed delivery in humans.

In addition, Brandão et al. [2011] reported a 44-yearold woman with bilateral streak gonads and moderate clitoromegaly, in whom the authors identified a homozygous missense variant p.Val505Ala (p.Val432Ala) of $M A M L D 1$. The patient was born to consanguineous parents. The variant MAMLD1 protein exerted higher in vi- 
tro transactivating activity for Hes 3 and Hes 7 promoters than the wildtype protein. These results provide the first indication that gain-of-function variants of MAMLD1 may be associated with ovarian dysfunction with partial masculinization. In this context, Baetens et al. [2017] reported that MAMLD1 is upregulated in the lymphocytes of individuals with 46,XX testicular DSD due to the increased MAMLD1 expression contributes to testicular development in genetic females. However, because no cases with 46,XX testicular DSD and pathogenic MAMLD1 variants have been reported so far, this speculation awaits further validation.

\section{Further Perspectives}

\section{MAMLD1 Function}

The role of MAMLD1 in sex development is not fully MAMLD1 transactivates its target genes without binding to DNA. Moreover, in vitro assays suggested that the function of MAMLD1 to promote testicular development is independent of Hes3 [Fukami et al., 2008]. Future studies on the oligogenicity of $M A M L D 1$-related DSD may provide a clue to understand the gene-gene interactions involving MAMLD1 in the developing gonads.

Although MAMLD1 was shown to reside in the nucleus in a speckled pattern [Fukami et al., 2008], the organelle which involves MAMLD1 has yet to be determined. In this regard, recent studies have demonstrated that mammalian cells contain a number of membraneless organelles both in the nucleus and in the cytoplasm [Hyman et al., 2014; Banani et al., 2017]. These membraneless organelles are created through liquid-liquid phase separation (LLPS) and facilitate various biochemical reactions including transcriptional regulation and RNA modification. In particular, recent studies have suggested that SOX9 is located in the paraspeckle, one of the major LLPS-associated nuclear organelles, and transactivates its target genes [Hata et al., 2008; Akiba et al., 2020]. These data indicate that LLPS contributes to testicular development by providing a functional platform for SOX9. The distribution pattern of MAMLD1 implies that it may also reside within an LLPS-associated membraneless organelle. p.Arg92Trp variant of NR5A1. These results imply that understood. For example, it remains unknown how

\section{MAMLD1 Intracellular Localization}

\section{Conclusions}

A number of rare $M A M L D 1$ variants have been identified in patients with 46,XY DSD. These patients typically exhibited hypospadias indicative of subnormal androgen production/function during the embryonic period. In addition, some $M A M L D 1$ variants have been linked to ovarian insufficiency in women. However, recent studies have raised the possibility that $M A M L D 1$ variants cause $46, \mathrm{XY}$ DSD and ovarian dysfunction as oligogenic disorders. Unsolved issues regarding MAMLD1 include the association between its variants and 46,XX testicular DSD and gene-gene interactions in the development of MAMLD1mediated DSD.

\section{Conflict of Interest Statement}

The authors have nothing to disclose.

\section{Funding Sources}

This study was funded by the Japan Society for the Promotion of Science (17H06428, 20H00539, 21K19283, and 21H02464), the Japan Agency for Medical Research and Development (21ek0109464h0002), the National Center for Child Health and Development (2019A-1), and the Takeda Science Foundation.

\section{Author Contribution}

M.F. drafted the manuscript. M.M. edited the text and prepared the figure and the table. T.O. edited the manuscript. All authors reviewed the final version of the manuscript.
References
Akiba K, Narumi S, Nishimura R, Kato-Fukui Y, Takada S, Hasegawa Y, et al. SOX9 is colocalized with paraspeckle protein NONO in cultured murine sertoli cells and features structural characteristics of intrinsically disordered proteins. Mol Reprod Dev. 2020;87:1124-5.

Baetens D, Stoop H, Peelman F, Todeschini AL, Rosseel T, Coppieters F, et al. NR5A1 is a novel disease gene for 46,XX testicular and ovotesticular disorders of sex development. Genet Med. 2017;19:367-76.

Banani SF, Lee HO, Hyman AA, Rosen MK. Biomolecular condensates: organizers of cellular biochemistry. Nat Rev Mol Cell Biol. 2017;18: 285-98.

Baxter RM, Arboleda VA, Lee H, Barseghyan H, Adam MP, Fechner PY, et al. Exome sequencing for the diagnosis of 46,XY disorders of sex development. J Clin Endocrinol Metab. 2015; 100:E333-44. 
Brandão MP, Costa EM, Fukami M, Gerdulo M, Pereira NP, Domenice S, et al. MAMLD1 (mastermind-like domain containing 1) homozygous gain-of-function missense mutation causing 46,XX disorder of sex development in a virilized female. Adv Exp Med Biol. 2011;707:129-31.

Camats N, Fernández-Cancio M, Audí L, Mullis PE, Moreno F, González Casado I, et al. Human MAMLD1 gene variations seem not sufficient to explain a $46, \mathrm{XY}$ DSD phenotype. PLoS One. 2015;10:e0142831.

Camats N, Flück CE, Audí L. Oligogenic origin of differences of sex development in humans. Int J Mol Sci. 2020;21:1809.

Chen Y, Thai HT, Lundin J, Lagerstedt-Robinson K, Zhao S, Markljung E, et al. Mutational study of the MAMLD1-gene in hypospadias. Eur J Med Genet. 2010;53:122-6.

De Lorenzi L, Genualdo V, Iannuzzi A, Di Meo GP, Perucatti A, Mancuso R, et al. Cytogenetic and genetic studies in a hypospadic horse (Equus caballus, $2 \mathrm{n}=64$ ). Sex Dev. 2010;4: 352-7.

Eggers S, Sadedin S, van den Bergen JA, Robevska G, Ohnesorg T, Hewitt J, et al. Disorders of sex development: insights from targeted gene sequencing of a large international patient cohort. Genome Biol. 2016;17:243.

Flück CE, Audí L, Fernández-Cancio M, Sauter KS, Martinez de LaPiscina I, Castaño L, et al. Broad phenotypes of disorders/differences of sex development in MAMLD1 patients through oligogenic disease. Front Genet. 2019; 10:746.

Fujisawa Y, Fukami M, Hasegawa T, Uematsu A, Muroya K, Ogata T. Long-term clinical course in three patients with MAMLD1 mutations. Endocr J. 2016;63:835-9.

Fukami M, Wada Y, Miyabayashi K, Nishino I, Hasegawa T, Nordenskjöld A, et al. CXorf6 is a causative gene for hypospadias. Nat Genet. 2006;38:1369-71.

Fukami M, Wada Y, Okada M, Kato F, Katsumata N, Baba T, et al. Mastermind-like domaincontaining 1 (MAMLD1 or CXorf6) transactivates the Hes 3 promoter, augments testosterone production, and contains the SF1 target sequence. J Biol Chem. 2008;283:552532.

Gaspari L, Paris F, Philibert P, Audran F, Orsini $\mathrm{M}$, Servant N, et al. Idiopathic partial androgen insensitivity syndrome in 28 newborn and infant males: impact of prenatal exposure to environmental endocrine disruptor chemicals? Eur J Endocrinol. 2011;165:579-87. https://doi.org/10.1530/EJE-11-0580.

Hata K, Nishimura R, Muramatsu S, Matsuda A, Matsubara T, Amano K, et al. Paraspeckle protein p54nrb links Sox9-mediated transcription with RNA processing during chondrogenesis in mice. J Clin Invest. 2008;118: 3098-108.
Hughes LA, McKay-Bounford K, Webb EA, Dasani P, Clokie S, Chandran H, et al. Nextgeneration sequencing (NGS) to improve the diagnosis and management of patients with disorders of sex development (DSD). Endocr Connect. 2019;8:100-10.

Hyman AA, Weber CA, Jülicher F. Liquid-liquid phase separation in biology. Annu Rev Cell Dev Biol. 2014;30:39-58.

Igarashi M, Wada Y, Kojima Y, Miyado M, Nakamura M, Muroya K, et al. Novel splice site mutation in MAMLD1 in a patient with hypospadias. Sex Dev. 2015;9:130-5.

Kageyama R, Ohtsuka T, Hatakeyama J, Ohsawa R. Roles of bHLH genes in neural stem cell differentiation. Exp Cell Res. 2005;306:343-8.

Kalfa N, Liu B, Klein O, Ophir K, Audran F, Wang $\mathrm{MH}$, et al. Mutations of CXorf6 are associated with a range of severities of hypospadias. Eur J Endocrinol. 2008;159:453-8.

Kalfa N, Cassorla F, Audran F, Oulad Abdennabi I, Philibert P, Béroud C, et al. Polymorphisms of MAMLD1 gene in hypospadias. J Pediatr Urol. 2011;7:585-91.

Kalfa N, Fukami M, Philibert P, Audran F, Pienkowski C, Weill J, et al. Screening of MAMLD1 mutations in 70 children with 46,XY DSD: identification and functional analysis of two new mutations. PLoS One. 2012;7:e32505.

Kon M, Suzuki E, Dung VC, Hasegawa Y, Mitsui T, Muroya K, et al. Molecular basis of nonsyndromic hypospadias: systematic mutation screening and genome-wide copy-number analysis of 62 patients. Hum Reprod. 2015;30: 499-506.

Laporte J, Guiraud-Chaumeil C, Vincent MC, Mandel JL, Tanner SM, Liechti-Gallati S, et al. Mutations in the MTM1 gene implicated in X-linked myotubular myopathy. ENMC International Consortium on Myotubular Myopathy. European Neuro-Muscular Center. Hum Mol Genet. 1997;6:1505-11.

Li L, Gao F, Fan L, Su C, Liang X, Gong C. Disorders of sex development in individuals harbouring MAMLD1 variants: WES and interactome evidence of oligogenic inheritance. Front Endocrinol. 2020a;11:582516.

Li L, Su C, Fan L, Gao F, Liang X, Gong C. Clinical and molecular spectrum of 46,XY disorders of sex development that harbour MAMLD1 variations: case series and review of literature. Orphanet J Rare Dis. 2020b;15:188.

Marozzi A, Manfredini E, Tibiletti MG, Furlan D, Villa N, Vegetti W, et al. Molecular definition of Xq common-deleted region in patients affected by premature ovarian failure. Hum Genet. 2000;107:304-11.

Metwalley KA, Farghaly HS. X-linked congenital adrenal hypoplasia associated with hypospadias in an Egyptian baby: a case report. J Med Case Rep. 2012;6:428.
Miyado M, Nakamura M, Miyado K, Morohashi K, Sano S, Nagata E, et al. Mamld1 deficiency significantly reduces mRNA expression levels of multiple genes expressed in mouse fetal Leydig cells but permits normal genital and reproductive development. Endocrinology. 2012;153:6033-40.

Miyado M, Miyado K, Katsumi M, Saito K, Nakamura A, Shihara D, et al. Parturition failure in mice lacking Mamld1. Sci Rep. 2015;5:14705.

Miyado M, Yoshida K, Miyado K, Katsumi M, Saito K, Nakamura S, et al. Knockout of murine Mamld1 impairs testicular growth and daily sperm production but permits normal postnatal androgen production and fertility. Int J Mol Sci. 2017;18:1300.

Nakamura M, Fukami M, Sugawa F, Miyado M, Nonomura K, Ogata T. Mamld1 knockdown reduces testosterone production and $\mathrm{Cy}$ p17a1 expression in mouse Leydig tumor cells. PLoS One. 2011;6:e19123.

Nazor KL, Altun G, Lynch C, Tran H, Harness JV, Slavin I, et al. Recurrent variations in DNA methylation in human pluripotent stem cells and their differentiated derivatives. Cell Stem Cell. 2012;10:620-34.

Ogata T, Fukami M, Wada Y. MAMLD1 (CXorf6) is a new gene for hypospadias. Clin Pediatr Endocrinol. 2008;17:87-93.

Ogata T, Sano S, Nagata E, Kato F, Fukami M. MAMLD1 and 46,XY disorders of sex development. Semin Reprod Med. 2012;30:410-6.

Ratan SK, Sharma A, Kapoor S, Polipalli SK, Dubey D, Mishra TK, et al. Polymorphism of 3' UTR of MAMLD1 gene is also associated with increased risk of isolated hypospadias in Indian children: a preliminary report. Pediatr Surg Int. 2016;32:515-24.

Richards S, Aziz N, Bale S, Bick D, Das S, GastierFoster J, et al. Standards and guidelines for the interpretation of sequence variants: a joint consensus recommendation of the American College of Medical Genetics and Genomics and the Association for Molecular Pathology. Genet Med. 2015;17:405-24.

Ruiz-Arana IL, Hübner A, Cetingdag C, Krude H, Grüters A, Fukami M, et al. A novel hemizygous mutation of MAMLD1 in a patient with $46, \mathrm{XY}$ complete gonadal dysgenesis. Sex Dev. 2015;9:80-5.

Switonski M, Payan-Carreira R, Bartz M, Nowacka-Woszuk J, Szczerbal I, Colaço B, et al. Hypospadias in a male (78,XY; SRY-positive) dog and sex reversal female (78,XX; SRY-negative) dogs: clinical, histological and genetic studies. Sex Dev. 2012;6:128-34.

Tsai TC, Horinouchi H, Noguchi S, Minami N, Murayama K, Hayashi YK, et al. Characterization of MTM1 mutations in 31 Japanese families with myotubular myopathy, including a patient carrying $240 \mathrm{~kb}$ deletion in $\mathrm{Xq} 28$ without male hypogenitalism. Neuromuscul Disord. 2005; 15:245-52. 